\title{
Effect of Ternary Additions to Structural Properties of NiTi Alloys
}

\author{
Navdeep Singh $^{a, 1}$, Anjana Talapatra ${ }^{b, 1}$, Anchalee Junkaew $^{c}$, Thien Duong ${ }^{b}$, Sean Gibbons ${ }^{d}$, \\ Shengyen $\mathrm{Li}^{d}$, Hassan $\mathrm{Thawabi}^{b}$, Emmi Olivos${ }^{e}$, Raymundo Arróyave ${ }^{b, d}$ \\ ${ }^{a}$ Department of Engineering Technology, University of Houston, USA, 77204 \\ ${ }^{b}$ Department of Mechanical Engineering, Texas A\&M University, USA, 77843 \\ ${ }^{c}$ NANOTEC, National Science and Technology Development Agency (NSTDA),Pathum Thani 12120, Thailand \\ ${ }^{d}$ Department of Materials Science and Engineering, Texas A\&M University, USA, 77843 \\ e Centro de Investigación de Materiales Avanzados (CIMAV), Monterrey, Nuevo Léon, México,
}

\begin{abstract}
In this work, the effects of small ternary additions to B2 NiTi structures was investigated through DFT calculations. The analysis considered deviations from stoichiometry arising from either simple substitution of host atoms in a given sublattice or from the formation of anti-sites. The calculations enabled the determination of the site preference of $\mathrm{X}$ ternary additions. Moreover, the results suggest that ternary additions located in the central region of the transition metal group across all periods tend to occupy $\mathrm{Ni}$ sites due to favorable X-Ti nearest neighbor (NN) interactions. This occupancy is achieved through substitution or through the generation of anti-site defects. On the other hand, ternary additions at both ends of a given transition metal row tend to occupy Ti sites due to favorable X-Ni NN interactions. Once site preferences are determined, the effect of alloying on the thermodynamic and mechanical properties of B2 NiTi-X structures are presented and trends are discussed.
\end{abstract}

Keywords: NiTi Alloys, Ab-initio calculations, Ternary Alloys, Structural Properties

\section{Introduction}

Shape Memory Alloys (SMAs) exhibit the so-called shape memory effect (SME)—large reversible and macroscopic shape change triggered by temperature changes - and superelasticity (SME) - large recoverable strain triggered by mechanical stress - as a result of reversible thermoelastic martensitic transformations [1]. Owing to this behavior, SMAs (particularly those based on the Ni-Ti system) have become the material class of choice in the biomedical industry as they have enabled a wide range of biomedical equipment and devices in orthopedics, neurology, cardiology and interventional radiology [2]. In the case of NiTi-based SMAs, the relevant structural transition typically involves the transformation from a high temperature cubic austenite phase (B2-type) to a low symmetry monoclinic phase (B19'), although other martensite structures (such as the so-called R-phase) may be stabilized depending on the chemistry and microstructure of the austenite $[3,4]$.

\footnotetext{
${ }^{1}$ Corresponding author. E-mail: nsingh8@uh.edu, anjanatalapatra@tamu.edu

Preprint submitted to Elsevier

October 22, 2015
}

(C) 2015. This manuscript version is made available under the Elsevier user license http://www.elsevier.com/open-access/userlicense/1.0/ 
Further development of SMAs has been driven by the aerospace and automotive industries due to their potential as compact, reliable solid-state actuators [5]. More recently, the oil and gas industry has explored the use of SMAs [6] as couplers and fasteners. Unfortunately, usage of SMAs is limited by their typically low (below $100{ }^{\circ} \mathrm{C}$ ) transformation temperatures. In the aerospace and automotive industries, in particular, design actuation temperatures are significantly higher than the operational limit of binary NiTi alloys [1]. Over the past decade, considerable effort has been invested in modifying the chemistry of NiTi-based SMAs (through ternary and quaternary substitutions of $\mathrm{Ni}$ and/or $\mathrm{Ti}$ ) to increase transformation temperatures [7], control hysteresis [8], and/or improve mechanical properties such as strength and corrosion resistance [9].

\subsection{Effects on martensitic transformation characteristics}

There is already considerable experimental evidence for the dramatic effect of ternary additions on the martensitic transformation temperatures as well as phase transformation sequence in NiTibased SMAs. Upon substitution of $\mathrm{Ni}$ by $\mathrm{Cu}$ at levels of about 10 at. \%, a two-stage martensitic transformation is seen [10], where the cubic B2 austenite transforms first to orthorhombic B19, followed by a transformation to the monoclinic martensite B19', contrary to the B2 $\rightarrow$ B19' typically observed in NiTi. Upon additions above 10 at. $\% \mathrm{Cu}$, the transformation reverts to the conventional single stage process. Addition of $\mathrm{Cu}$ also makes the martensitic start temperature $\left(M_{s}\right)$ [11] and pseudoelastic hysteresis $[12,13]$ less sensitive to composition changes and also prevents the precipitation of the metastable X-phase $\left(\mathrm{Ni}_{4} \mathrm{Ti}_{3}\right)$ [13]. Finally, the transformation hysteresis associated with the B2-B19 transformation in NiTi-Cu is between those of the B2-B19' and B2-R in $\mathrm{Ni}-\mathrm{Ti}[12]$.

Replacement of small amounts of of $\mathrm{Ti}$ by $\mathrm{Zr}$ in the $\mathrm{NiTi}$ alloy raises the $M_{s}$ temperature in NiTi-Zr SMAs [14]. Mulder et al. [15] reported $M_{s}$ temperatures above $120^{\circ} \mathrm{C}$ in NiTi-Zr alloys for $\mathrm{Zr}$ content above 10 at.\% and $\mathrm{Ni}$ content below 49.5 at \%. Addition of $\mathrm{Ag}$ to NiTi increases the austenitic transformation temperature $\left(A_{s}\right)$. The NiTi-Ag alloys also showed higher martensitic phase fractions than several other NiTi based alloys.

Potapov et. al. studied 8-20 at.\% Hf, NiTi-Hf alloys fabricated by melt spinning, paying attention to the $\mathrm{B} 19^{\prime} \rightarrow \mathrm{B} 2$ transformation at temperatures varying from 100 to $300{ }^{\circ} \mathrm{C}$, and found that the lattice parameter of the parent B2 phase increases with $\mathrm{Hf}$ addition while the transformation volume almost remains constant. The transformation temperatures as well as the transformation temperature intervals between start and finish temperatures were found to increase [16]. Studies on rapidly solidified NiTi-Zr and NiTi-Hf SMAs have been performed using calorimetric and mechanical measurements, with emphasis on microstructural features and their influence on the martensitic transformation. The results show that in Zr- and Hf-substituted alloys the microstructure resulting from the rapid solidification conditions strongly effects the characteristic transformation temperatures. Higher solubility of the alloying components, and the present of coherent precipitates and other secondary phases lead to a significant decrease in $\mathrm{M}_{s}$ [17]. On the other hand, Studies by Wojcik et. al. shows that addition of Hafnium up to 10 at.\% leads to increases in the transformation temperature by almost $100{ }^{\circ} \mathrm{C}[18]$.

One-way and two-way SME have been studied in DC-magnetron sputtered Ti-Ni, Ti-Ni-Pd and $\mathrm{Ti}-\mathrm{Pd}$ thin films. Research showed that by substituting Ni with $\mathrm{Pd}$ the transition temperatures could be increased from $32-38$ to $498-570{ }^{\circ} \mathrm{C}$ for Ti-Pd films [19]. Inter-diffusion of $\mathrm{Ni}$ and $\mathrm{Si}$ in NiTi thin films deposited on a silicon substrate and the resulting shape memory properties have been investigated and the resulting $\mathrm{Ni}_{x} \mathrm{Ti}_{y} \mathrm{Si}_{z}$ ternary alloys exhibited transition temperatures higher than NiTi SMAs [20]. 
The transformation behavior, shape memory effect and superelasticity of NiTi-V with equal substitution of $\mathrm{V}$ for both $\mathrm{Ti}$ and $\mathrm{Ni}$ have been studied and results show that adding 1-2 at. \% $\mathrm{V}$ resulted in a slight drop $\left(10^{\circ} \mathrm{C}\right)$ in a single-stage $\mathrm{B} 2 \rightarrow \mathrm{B} 19^{\prime}$ martensitic transformation. Solid solution strengthening of NiTi SMAs by V improves their superelastic and shape memory response, although the formation of $(\mathrm{Ti}, \mathrm{V})_{2} \mathrm{Ni}$ second-phase particles within the NiTi-V matrix absorb oxygen atoms to form the $(\mathrm{Ti}, \mathrm{V})_{4} \mathrm{Ni}_{2} \mathrm{O}$ oxide and in turn degrade the alloys' shape memory behavior [21].

\subsection{Effects on performance}

Up to $1.5 \mathrm{wt} \% \mathrm{Ag}$ increased the corrosion resistance of the alloys, but at higher silver levels the corrosion resistance showed a decrease [22]. Compressive strength is seen to improve dramatically at room temperature and higher temperatures by the substitution of $\mathrm{Ti}$ with $\mathrm{Al}$ [23]. This improvement in strength is partly due to the formation of $\mathrm{Ni}_{2} \mathrm{TiAl}$ (Heusler compound) precipitate which is coherent with the $\mathrm{NiTi}(\mathrm{B} 2)$ matrix. In particular, researchers reported that an addition of 8.4 at.\% $\mathrm{Al}$ showed an increase in compressive strength to $2400 \mathrm{MPa}$ (at room temperature) and 250 $\mathrm{MPa}\left(\right.$ at $1000{ }^{\circ} \mathrm{C}$ ) comparable with mid-grade superalloys U500/U700 and Rene95 respectively. Experiments with Co addition show that substitution of $\mathrm{Ni}$ by $\mathrm{Co}$ in NiTi improves the yield stress and work hardening coefficient between room temperature and about $380{ }^{\circ} \mathrm{C}$ [24]. Moreover, addition of 1-2 at.\% Co to NiTi increases the modulus, loading and unloading plateau by $30 \%$ as compared to the binary NiTi alloy [25].

Although not stated explicitly, studies suggest that the occupation of $\mathrm{V}$ on $\mathrm{Ti}$ sites yield a higher wear resistance, hardness and pseudoelastic behavior [21]. The ternary addition of Cr effects martensite transformation temperatures, $M_{s}$, as well as mechanical properties of off-stoichiometric NiTi alloys. $\mathrm{M}_{s}$ and $\mathrm{A}_{s}$ decrease with decreasing Ti concentration and change slightly when $\mathrm{Ti}$ concentration exceeds 52 at.\%, where the alloys are in the two phase region. Significantly, $\mathrm{M}_{s}$ and $\mathrm{A}_{s}$ decreased with increasing $\mathrm{Cr}$ content at a constant $\mathrm{Ti}$ concentration. It was suggested that $\mathrm{Cr}$ atoms will substitute $\mathrm{Ni}$ sites preferably if only $\mathrm{M}_{s}$ changes are observed. Based on the characteristic stress-strain curves presented, the stress induced martensitic transformation (SIMT) occurred at $-196^{\circ} \mathrm{C}[24]$.

\subsection{Effects on biocompatibility}

Owing to their biocompatibility, NiTi-based SMAs are very useful in medical applications. SMA's have a high work output, which is necessary for minimally invasive instruments $[26,27]$ and are superelastic, which makes them suitable for implants such as coronary stents [28]. Enhanced antibacterial properties, which can be achieved by addition of Ag, can significantly broaden their range of application. Silver is known for its antibacterial properties and Ag-alloy coatings are effectively used against a number of bacteria [29, 30]. Moreover, addition of $\mathrm{Ag}$ also improves the corrosion resistance of NiTi SMAs which makes them suitable for orthodontic applications [22]. $\mathrm{NiTi}$-Co are being considered for potential use since their superelasticity allows for durable, lower profile and less intrusive medical devices. NiTi-Co is also comparable to NiTi in terms of fatigue, corrosion resistance and biocompatibility. In NiTi-Mo alloys corrosion resistance can be improved by increasing an amount of Mo leading to improvement in biocompatibility [31].

\subsection{Prior computational studies on NiTi-X alloying behavior}

Bozzolo et. al. [32, 33] developed an approach based on quantum approximations, coined the Bozzolo-Ferrante-Smith (BFS) method, for analyzing SMA properties. In particular, Bozzolo focused on ternary bridge $\mathrm{Ni}_{50-y} \mathrm{Ti}_{50} \mathrm{X}_{y}$ (i.e., where $\mathrm{X}=\mathrm{Fe}, \mathrm{Pd}, \mathrm{Pt}, \mathrm{Au}, \mathrm{Al}, \mathrm{Cu}, \mathrm{Zr}$, and Hf). The 
BFS method is based on the assumption that the energy of formation of a given atomic configuration can be quantified in terms of individual atomic contributions that account for lattice strain energy as well as chemical effects. The strain energy contribution can be obtained from the cohesive, structural and mechanical properties of the pure elements, while the chemical contribution accounts for the energetics of so-called pure and mixed bonds. In essence, the BFS method provides a very effective and computationally economical approach to investigate the energetics of alloying. One must point out, however, that this method is only indirectly related to first-principles calculations and some subtle bonding effects that cannot be simply separated into mechanical and chemical effects may not be captured.

In the case of their study of ternary X substitutions in NiTi, through the use of the BFS method, Bozzolo and collaborators were able to derive the lattice parameter, bulk modulus and energy of formation for the intermediate states in the (B2) transition NiTi to XTi. The substitutional elements considered through the BFS method in the work by Bozzolo and collaborators [32-34] were Au, Pt, Ir, Os, Re, W, Ta, Ag, Pd, Rh, Ru, Tc, Mo, Nb, Zr, Zn, Cu, Co, Fe, Mn, V, Sc, Si, Al and Mg. In a subsequent work by Mosca et. al. [35], the BFS method was used to assess the behavior of ternary and quaternary additions to NiTi (i.e. ternary additions to NiTi and quaternary additions to NiTiPd, NiTiPt, and NiTiHf).

\subsection{Overview of the present work}

In this work, we present a systematic investigation of the effects of ternary additions - in the dilute limit-on the thermodynamic and mechanical properties of B2 NiTi-X alloys. A variety of experiments and simulations have been carried out to study the effect of a ternary element on the shape memory, tensile and performance properties of NiTi-based SMAs. The purpose of this work is to carry out a systematic high-throughput computational study of the effect of ternary alloying on equiatomic NiTi alloys. The energetics as well as physical properties of $34 \mathrm{NiTi}$ ternary alloys have been investigated and presented. The ternary elements considered were: $\mathrm{Mg}, \mathrm{Al}, \mathrm{Si}, \mathrm{Sc}, \mathrm{Ti}$, V, Cr, Mn, Fe, Co, Ni, Cu, Zn, Ga, Ge, Y, Zr, Nb, Mo, Tc, Ru, Rh, Pd, Ag, Cd, In, Sn, Sb, Hf, Ta, W, Re, Os, Ir, Pt and Au.

The paper is organized as follows: first, existing experimental evidence for the effect of ternary additions on the martensitic transformation, mechanical and other functional properties is discussed; we follow the discussion by presenting the methods used; then we analyze the site preference for ternary additions and the calculations are compared to results from other computational approaches; finally, the effect of ternary additions on the thermodynamic and mechanical properties of B2 NiTi$\mathrm{X}$ alloys is discussed.

\section{Computational Approach}

First principles methods were used to calculate the energetic and structural properties of NiTi$\mathrm{X}$ SMA alloys with small ternary additions. The concentration of ternary additions (hereafter referred to as element X) has been limited to $3.125 \%$. The initial supercell structures have been modeled as special quasirandom structures (SQS) [36] that minimized the difference in the pair and higher-order correlation functions between the simulated structures and true random alloys, up to six coordination shells. The 'mcsqs' module of the ATAT package $[37,38]$ was used to construct the structures - see Fig. 1. In this study, we have considered a supercell structure consisting of 32 atoms with $15 \mathrm{Ni}(\mathrm{Ti}), 16 \mathrm{Ti}(\mathrm{Ni})$ and one $\mathrm{X}$ element in a $\mathrm{Ti}(\mathrm{Ni})$ rich alloy. The configurations were ensured to be as close to a random solution as possible by constructing SQS structures. 
Figure 1: (Top) Image shows the stacking of stochiometric NiTi B2 unit cells to form a supercell. The orientation and location of the (110) plane is shown in light color. The top right image show the front view of the (110) plane and the arrangement of atoms in the plane. The rectangles demonstrate the motif for $\mathrm{Ni}$ and $\mathrm{Ti}$ atomic arrangement. These supercells are for explanation only, and have not been used to perform the calculations. (Below) SQS structures, generated and used to perform the calculations, for conf1 and its (010) plane, and conf2 and its (111) plane for $\mathrm{Ni}_{15} \mathrm{Ti}_{16} \mathrm{X}$ alloy. For $\mathrm{Ni}_{16} \mathrm{Ti}_{15} \mathrm{X}$ alloy, these configurations have been obtained by replacing $\mathrm{Ni}(\mathrm{Ti})$ atoms with the $\mathrm{Ti}(\mathrm{Ni})$ atoms. The rectangles exhibit how the motif in the (110) plane in the B2 supercell above rearranges in the SQS structures, on (010) plane for conf1 and (111) plane (exact plane indices are (1.015 1.021 1)) for conf2. The blue (red) rectangles demonstrate $\mathrm{Ti}(\mathrm{Ni})$ atom surrounded by the $\mathrm{Ni}(\mathrm{Ti})$ atoms. For Pt alloy system (element $\mathrm{X}$ is $\mathrm{Pt}$ ), the coordinates of the conf1 SQS unit cell are $\mathrm{a}=7.323 \AA, \mathrm{b}=4.224 \AA$, and $\mathrm{c}=14.005 \AA$ while for conf2 the coordinates of SQS unit cell are $\mathrm{a}=\mathrm{b}=6.688 \AA$, and $\mathrm{c}=9.846 \AA$. In conf1, the distance between the nearest $\mathrm{Pt}$ atoms is 4.224 Åwhile in conf2 the nearest distance is $6.688 \AA$. These images have been generated using VESTA software [39]. Please refer to text for further description of configurations.

With the structures in place, the respective systems were evaluated within the framework of Density Functional Theory (DFT) [40], as implemented in the Vienna ab-initio simulation package (VASP) [41], applying the generalized gradient approximation (GGA) and the Perdew - Burke Ernzerhof (PBE) functional [42]. The electronic configurations of the appropriate elements were determined using the projector augmented-wave (PAW) pseudo-potentials formalism [43, 44], while 
Brillouin zone integrations were performed using a Monkhorst-Pack mesh [45] with at least 3000 k-points per reciprocal atom. A cutoff energy of $478 \mathrm{eV}$ was used and spin polarizations were accounted for as well.

Optimal structures were obtained as follows: first, a volume-only relaxation was carried out. The volume-relaxed structures were then subjected to structural optimizations in which the volume and shape were allowed to vary. Finally, full relaxations were carried out, including volume, shape and ionic positions as degrees of freedom - using order-one Methfessel-Paxton smearing method [46]. All relaxations were carried out until changes in total energy between relaxation steps was within $1 \times 10^{-6} \mathrm{eV}$. After the relaxation, the electronic structure of the optimized structures was calculated once more using a self-consistent static calculation scheme using the tetrahedron smearing method with Blöchl corrections [47].

The elastic constants were estimated through the stress-strain approach [48, 49] where a set of strains $\left(\varepsilon=\varepsilon_{1}, \varepsilon_{2}, \varepsilon_{3}, \varepsilon_{4}, \varepsilon_{5}, \varepsilon_{6}\right)$ is imposed on a crystal structure. The deformed lattice vectors are then defined as:

$$
\bar{A}=A\left|\begin{array}{ccc}
1+\varepsilon_{1} & \frac{\varepsilon_{6}}{2} & \frac{\varepsilon_{5}}{2} \\
\frac{\varepsilon_{6}}{2} & 1+\varepsilon_{2} & \frac{\varepsilon_{4}}{2} \\
\frac{\varepsilon_{5}}{2} & \frac{\varepsilon_{4}}{2} & 1+\varepsilon_{3}
\end{array}\right| .
$$

A set of stresses $\left(\sigma=\sigma_{1}, \sigma_{2}, \sigma_{3}, \sigma_{4}, \sigma_{5}\right.$ and $\left.\sigma_{6}\right)$ for the deformed crystals is generated, which is calculated using density functional theory (DFT) methods as described in $[48,49]$. From the $n$ set of strains and the resulting stresses, elastic constants are calculated based on Hooke's law, as shown below.

$$
\left|\begin{array}{ccc}
C_{11} & \cdots & C_{16} \\
\vdots & & \vdots \\
C_{61} & \cdots & C_{66}
\end{array}\right|=\left|\begin{array}{ccc}
\varepsilon_{1,1} & \cdots & \varepsilon_{1, n} \\
\vdots & & \vdots \\
\varepsilon_{6,1} & \cdots & \varepsilon_{6, n}
\end{array}\right|^{-1}\left|\begin{array}{ccc}
\sigma_{1,1} & \cdots & \sigma_{1, n} \\
\vdots & & \vdots \\
\sigma_{6,1} & \cdots & \sigma_{6, n}
\end{array}\right|
$$

For the elastic constant calculations, the ionic positions were relaxed while leaving the lattice shape and volume invariant. These calculations were followed by a static calculation using orderone Methfessel-Paxton smearing method and an auxiliary FFT grid to ensure maximum accuracy in the calculation of interatomic forces. Convergence criteria ensured that calculated elements of elastic constant tensor changed within a few GPa when varying the magnitude of the lattice strain from 1 to $3 \%$.

From these elastic constants, various elastic properties have been calculated using the Voigt and Reuss approximations and Voigt-Reuss-Hill averaging (see table 1). The properties under consideration are: the bulk modulus (B), the shear Modulus (G), the Young's modulus (E), Poisson's ratio $(\nu)$, and the Debye temperature $\left(\Theta_{D}\right)$.

\section{Results and Discussion}

\subsection{Site Preference}

The effect of addition of ternary elements to the stoichiometric NiTi alloy has been studied for $\mathrm{Ni}$ and $\mathrm{Ti}$ rich compositions. The ternary atoms replace either the $\mathrm{Ni}$ or $\mathrm{Ti}$ atoms in the stochiometric NiTi alloy, depending upon the experimental conditions, type of ternary atom etc., to create $\mathrm{Ti}$ or $\mathrm{Ni}$ rich alloys. In the present study $\mathrm{Ni}(\mathrm{Ti})$ rich alloys, $\mathrm{Ni}_{16} \mathrm{Ti}_{15} \mathrm{X}\left(\mathrm{Ni}_{15} \mathrm{Ti}_{16} \mathrm{X}\right)$, have been fashioned by replacing one of the $\mathrm{Ti}(\mathrm{Ni})$ atom with ternary atom, $\mathrm{X}$ (hereafter the Ni rich compositions will be referred to as $\mathrm{NiR}$ and $\mathrm{Ti}$ rich as $\mathrm{TiR}$ ). We have assumed that all the austenite 
Table 1: Formulas used in Calculations of Elastic Properties [50, 51]

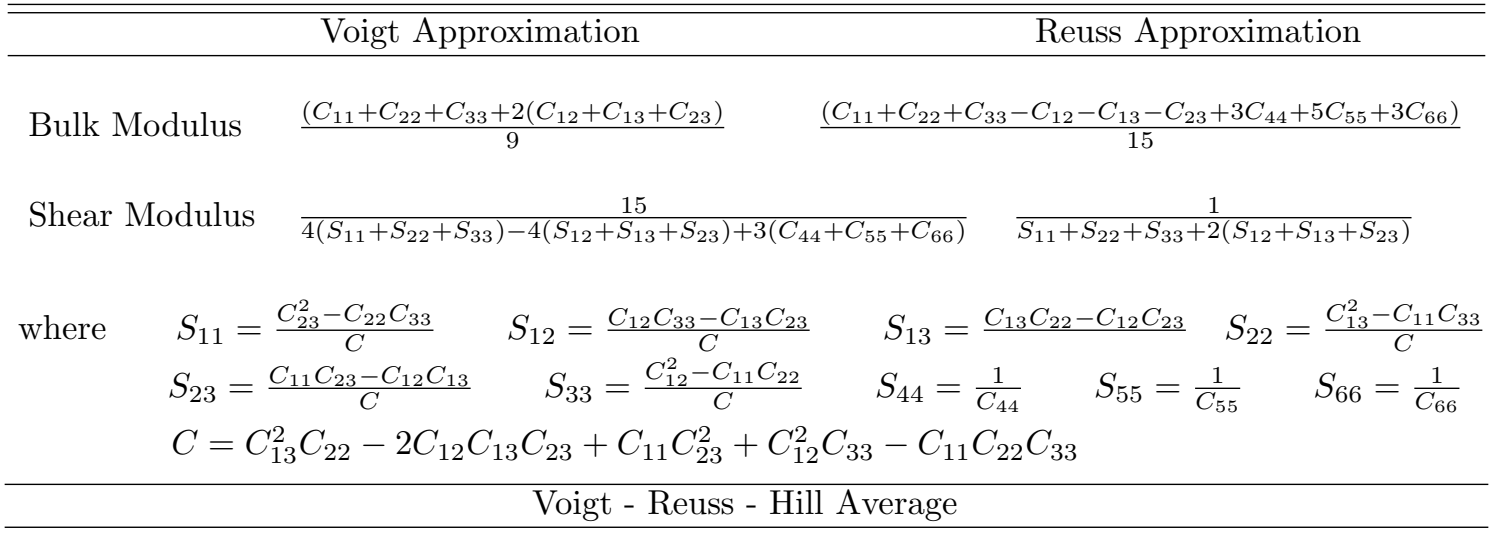

$\begin{array}{llll}\text { Bulk Modulus } & B=\frac{1}{2}\left(B_{V}+B_{R}\right) & \text { Transverse Wave Velocity } & v_{t}=\left(\frac{G}{\rho}\right)^{1 / 2} \\ \text { Shear Modulus } & G=\frac{1}{2}\left(G_{V}+G_{R}\right) & \text { Longitudinal Wave Vel. } & v_{l}=\left(\frac{3 B+4 G}{3 \rho}\right)^{1 / 2} \\ \text { Young's Modulus } & E=\frac{9 B G}{3 B+G} & \text { Sound Velocity } & v_{m}=\left[\frac{1}{3}\left(\frac{2}{v_{t}^{3}}+\frac{1}{v_{l}^{3}}\right)\right]^{-1 / 3} \\ \text { Poisson's Ratio } & \nu=\frac{3 B-E}{6 B} & \text { Debye Temperature } & \Theta_{D}=\frac{h}{k}\left[\frac{3 n}{4 \pi}\left(\frac{N_{A} \rho}{M}\right)\right]^{1 / 3} v_{m}\end{array}$

(high temperature) structures will be of type B2 even after the addition of a ternary element. This assumption is reasonable as the site occupancies were studied in the dilute limit. The site preference of the X element (whether in the $\mathrm{Ni}$ or Ti site) is decided based on the ground state energy of the system. Hence, for each composition (NiR or TiR) we have two types of configurations. The atomic structures for these compositions are shown in Fig. 1.

In the first configuration (hereafter referred to as conf1), for TiR (NiR) composition, $\mathrm{Ni}(\mathrm{Ti}$ ) and $\mathrm{X}$ atoms occupy one site (say, $0.0,0.0,0.0)$ and $\mathrm{Ti}(\mathrm{Ni})$ atoms sits on the other site, $\left(\mathrm{Ni}_{15}, \mathrm{X}\right),\left(\mathrm{Ti}_{16}\right)$ $\left(\left(\mathrm{Ti}_{15}, \mathrm{X}\right), \mathrm{Ni}_{16}\right)$. In the case of Ni-Ti-Pt, the $\mathrm{X}$-X distance is $4.224 \AA$, large enough to to discourage formation of $\mathrm{X}-\mathrm{X}$ atomic chains. In the second configuration (hereafter referred to as conf2), for TiR (NiR) alloy, the $\mathrm{X}$ atom sits on the $\mathrm{Ti}(\mathrm{Ni})$ site while one $\mathrm{Ti}(\mathrm{Ni})$ atom occupies the $\mathrm{Ni}(\mathrm{Ti})$ site, $\left(\mathrm{Ni}_{15}, \mathrm{Ti}\right),\left(\mathrm{Ti}_{15}, \mathrm{X}\right)\left(\left(\mathrm{Ti}_{15}, \mathrm{Ni}\right),\left(\mathrm{Ni}_{15}, \mathrm{X}\right)\right)$. X-X distance in Ni-Ti-Pt for conf2 is $6.688 \AA$. Since all structures were completely relaxed before calculation of properties, the actual X-X distances for both configurations will vary for different X, but will be close to $4.4 \AA$ for conf1 and 6.6 Åfor conf2.

We consider either the occupation - by substitution — of an available site (due to depletion of Ti or $\mathrm{Ni}$ ) or the occupation of a site made available through the formation of an anti-site defect. For example: a Ni-rich NiTi-X alloy is generated by the elimination of one (or more) Ti atoms. The X addition could occupy the available Ti site or could occupy the Ni site, through a generation of an anti-site defect where $\mathrm{Ni}$ migrates to the Ti lattice and the available $\mathrm{Ni}$ site is then occupied by X. At the ground state, occupancy is determined solely by the energetics of the competition between 
Figure 2: Site Preference of X element in Ni-Ti-X. The upper row (TiR) indicates site preference for Ti-rich compositions and the lower row (NiR) for Ni-rich compositions respectively.

'normal' and anti-site-mediated occupancy. At finite temperatures, however, one must consider the entropic differences between competing configurations [52]. In this work, we consider highly dilute systems where the entropy differences between configurations are likely to be small so we assume that enthalpic effects dominate occupation behavior.

Calculations were carried out for 34 ternary elements, viz : $\mathrm{Mg}, \mathrm{Al}, \mathrm{Si}, \mathrm{Sc}, \mathrm{Ti}, \mathrm{V}, \mathrm{Cr}, \mathrm{Mn}, \mathrm{Fe}$, Co, Ni, Cu, Zn, Ga, Ge, Y, Zr, Nb, Mo, Tc, Ru, Rh, Pd, Ag, Cd, In, Sn, Sb, Hf, Ta, W, Re, Os, $\mathrm{Ir}, \mathrm{Pt}$ and $\mathrm{Au}$. These elements include transition metals as well as $\mathrm{Mg}, \mathrm{Al}$ and Si. The supercell structures have been optimized to obtain the ground state energies of each configuration for both compositions.

The site preference was determined by comparing the formation energies of $\mathrm{Ni}_{x} \mathrm{Ti}_{y} \mathrm{X}_{z}$ alloys with competing configurations:

$$
\Delta E_{f}\left(N i_{x} T i_{y} X_{z}\right)=E\left(N i_{x} T i_{y} X_{z}\right)-x E(N i)-y E(T i)-z E(X)
$$

with the pure elements existing in their ground state (at $0 \mathrm{~K}$ ).

The configuration with lower ground state energy for each composition indicates the site preference of element X. For example, in case of Ti rich alloy, element X being Platinum (Pt), conf1 and conf2 have ground state energies of $-231.53 \mathrm{eV}$ and $-231.24 \mathrm{eV}$ respectively. Since conf1 is lower in energy (more negative), this means that Pt prefers to go to the Ni site.

From this analysis, the site preference for each atom is depicted through the use of color coding in Figure 2. The yellow color indicates a preference for the Ni site, while light blue indicates a preference for the Ti site. It is seen that groups 6-9 show an exclusive preference for the Ni site. 
This essentially means that for these alloying elements, $\mathrm{X}$ ternary additions prefer to be in a local environment in which they have $\mathrm{Ti}$ as its nearest neighbor. In the case of Ni-rich compositions (rather Ti-poor), this is achieved through the generation of an anti-site Ni defect. Using simple rigid band arguments, one could infer that the preference for $\mathrm{X}$-Ti, rather than $\mathrm{X}-\mathrm{Ni}$ interactions is due to the increase in stability of the structure upon filling of the Ti-X d-states. On the other hand, one can see that at both ends of the transition columns there is a tendency for Ti site preference, suggesting more energetically favorable $\mathrm{Ni}-\mathrm{X}$ nearest neighbor bonds.

Bozzolo et. al carried out extensive site-preference calculations for the Ni-Ti-X ( $\mathrm{X}=\mathrm{Pd}, \mathrm{Pt}$, $\mathrm{Al}, \mathrm{Fe}, \mathrm{Cu}, \mathrm{Au}, \mathrm{Zr}$ and $\mathrm{Hf}$ ) systems using the BFS method for alloys [34] which paved the way for further work calculating the lattice parameters, formation energies and bulk modulus for Pd, Pt, $\mathrm{Al}, \mathrm{Fe}, \mathrm{Cu}$ and $\mathrm{Au}$ additions [33]. These predictions agree appreciably with the site-preferences predicted in this work. Bozzolo et. al. [34] for example reports that in NiTi-Fe alloys Fe tends to occupy Ni sites in Ti-rich alloys, with no clear preference for either site in the case of NiTi-Fe Ni-rich alloys. In the same study, Bozzolo reports that $\mathrm{Al}, \mathrm{Au}$ and $\mathrm{Cu}$ tend to occupy the available sites through simple substitution, that is: the formation of anti-site defects is not favored on either side of the stoichiometric line. With the exception of $\mathrm{Cu}$, our results are consistent with those calculations as well as with earlier work by the same group [34]. Our results also agree with Bozzolo's Monte Carlo simulations that suggest that in NiTi-(Hf,Zr),Hf and Zr tend to occupy the Ti sites.

The formation energies for the preferred configurations for TiR and NiR alloys are shown in figures 3 and 4 respectively. The results show that most of the elements near the Ni atom prefer the Ni sites, while elements in close proximity to $\mathrm{Ti}$ on the periodic table prefer the $\mathrm{Ti}$ atomic sites. This can be due to the comparable size of the ternary additions to the host atoms. From the figure we see that the overall trend is an increase in the energy of formation as we go from the left to the right of the periodic table and also as we move down the periodic table in the same column. This can also be associated with the previous analysis of the site preference as it seems that Ti-X nearest neighbor interactions contribute significantly to the thermodynamic stability of NiTi-X ternary alloys. These trends and the corresponding values of formation of energy are in good agreement with the results reported by Bozzolo [33]. Unfortunately, to the best of the authors' knowledge there are no experiments evaluating the formation energy of NiTi-X alloys that could be used to validate the calculations.

\subsection{Structural Properties}

Having established the site occupancy in NiTi-X alloys, we proceeded to calculate their structural (i.e. mechanical) properties according to the procedure outlined in Section 2. As a benchmark of the accuracy of the methods used, we calculated the mechanical properties of NiTi stoichiometric SMAs and compared them with prior computational and experimental results, as shown in Table 2. Overall, there is acceptable agreement between our current calculations and prior work. Our calculations for the elastic constants agrees rather well - within $10 \mathrm{GPa}$ or less - with the computational work by Ye [53] and with the single crystal measurements by Mercier and collaborators [56] although these experimental measurements were done at finite temperatures (approximately $330 \mathrm{~K}$ ) and cannot be compared directly to the $0 \mathrm{~K}$ calculations.

According to our calculations, $C_{11}-C_{12}$ (twice the shear constant C') is positive, with values rather close $(24 \mathrm{GPa})$ to the measured ones $(33 \mathrm{GPa})$. Other theoretical works yield similar results (24 [53], 18 [54] and 40 [55] GPa). The small values of the shear constant are an indication of metastability (if not complete instability) against lattice distortions and indeed Huang et al. [54] found that the phonon structure for this system is unstable, with the most pronounced phonon 
instability localized around the $\mathrm{M}$ point and midway the $\Gamma-R$ direction of the Brillouin zone. The B2 lattice is likely to become entropically stabilized at finite temperatures due to anharmonic phonon-phonon interactions [54].

Here we would like to call into attention the work by Wagner and Windl [59] that contrary to the calculations reported in Table 2 reports that $C_{11}-C_{12}$ is negative. The results in this case were arrived at through the so-called strain-energy' method, which is based relating the curvature of energy vs deformation curves to linear combinations of the elastic constants. Using this approach, Wagner and Windl [59] reported that $C_{11}-C_{12}$ was negative (-30 GPa). In principle, a negative value for $C_{11}-C_{12}$ is consistent with the fact that the structure is dynamically unstable [54]. The discrepancy with respect to our calculations and those of Huang et al. could be ascribed to the different methods used. The 'stress-strain' method used by us may be yielding a positive shear constant due to the fact that we are only sampling the harmonic region of the energy landscape, while the 'strain-energy' approach by Wagner and Windl sample its non-harmonic - i.e. with negative curvature - region. Similar arguments are given by Hatcher et al. [60] who also report a positive shear constant. The phonon calculations by Huang report long wavelength stable acoustic modesthose responsible for the elastic constant tensor $\mathrm{C}_{i j}$-along the $\Gamma-X$ direction, which is the one directly associated with simple shear along the [110] direction. The $\mathrm{M}$ point on the other hand is associated with more complicated and non-symmetric atomic displacements [54], suggesting that signatures of instability would be more readily identified through lattice deformations more complex than the simple strains necessary to calculate the elastic constants. We would like to add that, to the best of our knowledge, no other theoretical work before [53-55] or after [60,61] Wagner and Windl [59] report negative shear constants and further systematic studies to elucidate the reason for the observed discrepancy are necessary.

Table 2 and the preceding discussion provide an indication of the validity of the methods used to calculate the elastic constants of ternary NiTi-X alloys. Following the success of the numerical procedure, the elastic properties for the TiR and NiR ternary alloys has been calculated. In this 
section the structural properties have been calculated exclusively for the preferred configuration. To the best of the authors knowledge, no systematic experimental data set on the effect of ternary alloying additions to NiTi-X alloys has been published. The major goal of this exercise is to determine trends that can be used to elucidate some of the alloying effects on the mechanical properties of NiTi-X alloys that have been observed experimentally, as briefly outlined in the introductory part of the present paper. We also hope that these trends could inspire the generation of alloy design guidelines that can help experimentalist in selecting the right substitution element depending on the properties of interest.

The computed properties are shown in figures 3 and 4 for Ti rich and Ni rich alloy systems. The addition of a ternary element increases the bulk modulus as compared to the NiTi binary system except for $\mathrm{Mg}, \mathrm{Si}, \mathrm{Ga}, \mathrm{Sb}$ and $\mathrm{Hf}$. These results are in fair agreement with the bulk moduli computed by Bozzolo et. al. [33], although they report that $\mathrm{Cu}, \mathrm{Zr}, \mathrm{Al}$, Hf tend to lower the bulk modulus as their amount increases while our calculations suggest that these elements do not change the bulk modulus in a significant manner. It is also worth considering the fact that our calculations correspond to relatively dilute alloying and further research must be carried out at significantly higher concentrations in order to elucidate composition effects in a more systematic manner. Our results indicate that $\mathrm{Pd}, \mathrm{Pt}$, and Fe increase the bulk modulus beyond the numerical uncertainties in the calculation - estimated to be on the order of 3-5 GPa. This is also consistent with the increased thermodynamic stability of $\mathrm{NiTi}-\mathrm{X}(\mathrm{Pd}, \mathrm{Pt}, \mathrm{Fe})$ alloys. An increase in bulk modulus indicates that $\mathrm{X}$ additions in general tend to increase the cohesive energy of NiTi-X structures. The Young's modulus and shear modulus, similar to the bulk modulus, increase with the addition of the ternary element with the exceptions of $\mathrm{Al}$ and $\mathrm{Si}$.

While the underlying physical principles for the effect of composition on the transformation temperatures of SMAs remains to be completely elucidated [62], there are currently several theories that have attempted to address this issue. Ren and Otsuka [63] used Landau theory to establish that martensitic transformations dominated by basal shear and shuffles occur at a critical shear constant, ' $\mathrm{c}$ ', which in turn is (roughly) independent of composition for a given nominal chemical system. By contrast, chemistry and temperature strongly affect ' $c$ '. They used this model to rationalize the fact that $\mathrm{Cu}$-based SMAs tend to show a higher $\mathrm{M}_{s}$ when the shear constant is lower. Extension to Ni-Ti based systems seemed to agree in the case of SMAs with low $\mathrm{M}_{S}$, although the analysis did not hold for high $\mathrm{M}_{s}$ systems.

The calculations of $C_{11}-C_{12}$ may lend an insight into the stability of the $B 2$ phase to shear and other martensitic transformation inducing deformations. As per the argument by Zarinejad and Liu [64], lower $C_{11}-C_{12}$ may result in higher transformation temperatures. Comparing with the extensive compilation by Zarinejad and Liu [64] it is not possible at this time to establish the validity of the reasoning, although the experimental data suggests that the most effective ternary additions at increasing $M_{s}$ are $\mathrm{Hf}, \mathrm{Zr}, \mathrm{Pt}$ and $\mathrm{Pd}$ and in our calculations those ternary additions generally result in a $C_{11}-C_{12}$ close to the lower range of the calculations.

There are, however, many exceptions to this trend, suggesting that the transformation temperature is the result of more subtle and complex aspects of the energy landscape around the austenite-martensite transformation. In fact, Frenzel et al. [62] experimentally investigated a wide range of NiTi-X SMAs and rationalized the strong compositional dependence of $\mathrm{M}_{s}$ in terms of enthalpy differences between austenite and martensite as measured by DSC as well as the influence of anti-site defects on the lattice distortion in near stoichiometric NiTi-X B2 cubic austenite. Smaller latent heats for transformation, $\Delta \mathrm{H}$, were generally correlated, albeit not perfectly, with lower $\mathrm{M}_{s}$. As shown in Fig. 5, other things remaining equal (i.e. entropy), compositional changes that reduce 
Figure 3: Energy of formation $(\delta E)$ in meV/atom and Debye temperature $\left(T_{D}\right)$ and Elastic constants: Bulk Modulus $(\mathrm{K})$, Elastic modulus (E), Shear Modulus (G), $C_{11}-C_{12}$ in GPa and Poisson's ratio ( $\nu$ ) for $\mathrm{Ni}_{15} \mathrm{Ti}_{16} \mathrm{X}(\mathrm{TiR})$. 
Figure 4: Energy of formation $(\delta E)$ in meV/atom and Debye temperature $\left(T_{D}\right)$ and Elastic constants: Bulk Modulus $(\mathrm{K})$, Elastic modulus (E), Shear Modulus (G), $C_{11}-C_{12}$ in GPa and Poisson's ratio $(\nu)$ for $\mathrm{Ni}_{16} \mathrm{Ti}_{15} \mathrm{X}(\mathrm{NiR})$. 
the enthalpy difference between austenite and martensite would necessarily result in a lowering of the temperature at which both phases have equal Gibbs energies, $\mathrm{T}_{0}$. Note that while $\mathrm{T}_{0}$ is related to $\mathrm{M}_{s}$, the latter is the result of barriers associated to the transformation that otherwise would occur at $\mathrm{T}_{0}$.

Finally, in the figures we report the Debye temperature, which corresponds to the frequency at which the wavelength of the phonon frequency equals the lattice parameter of the unit cell. For frequencies higher than this cutoff value, the vibration becomes independent of the lattice. The Debye cut off frequency or temperature signals a distinct barrier between the collective thermal lattice vibration and independent thermal lattice vibration and corresponds to the temperature at which all thermal phonon modes are excited. With the exceptions of $\mathrm{Al}, \mathrm{Pt}, \mathrm{Pd}$ and $\mathrm{Au}$; ternary alloying of equiatomic NiTi results in an increase in the Debye temperature, although Zr and $\mathrm{Hf}$ additions do not increase it significantly. Interestingly, these element additions tend to result in NiTi-X alloys with the highest transformation temperature.

\section{Conclusions}

In this systematic study of the effect of ternary alloying on the equiatomic NiTi B2 alloy, atomistic calculations were carried out to document the variation in the energetics, elastic properties and acoustic properties of $N i_{0.5} T i_{0.5}$ due to an impurity element. Owing to the complex nature of these alloys and the lack of literature on systematic studies, especially computational studies, this work fills in a gaping void in the framework for the future development of these alloys. While there are very clear systematics in the trends for cohesive energies (manifested in terms of bulk modulus), formation enthalpy and site occupancies, the trends are less clear in the case of the calculations for the mechanical properties (bulk modulus, Young's modulus, shear modulus, shear constant, etc).

More importantly, there remains a significant gap in our understanding of the effect of alloying elements on $\mathrm{M}_{S}$. While it has been suggested that changes in elastic constants (shear elastic

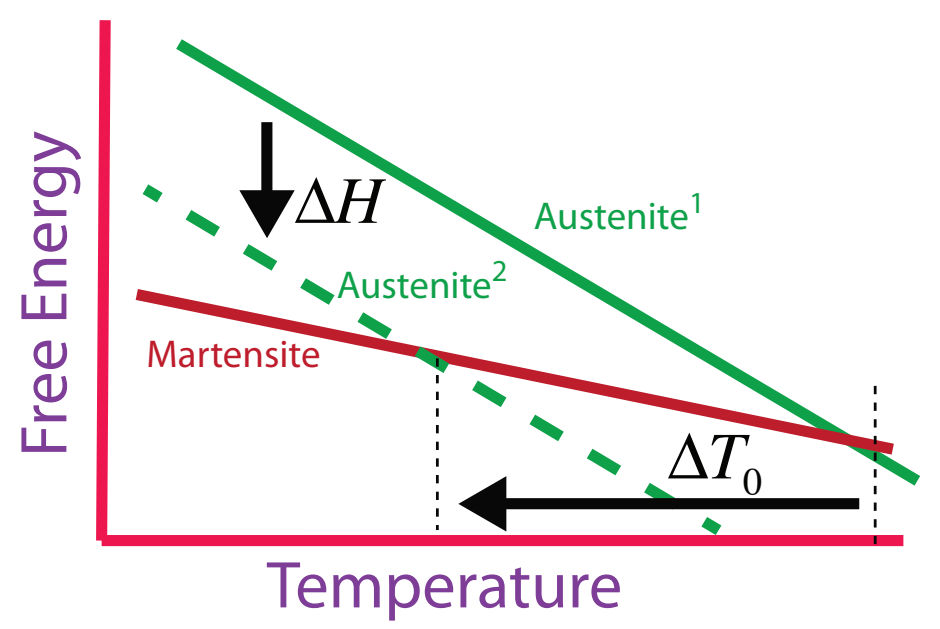

Figure 5: Schematic representation of the effect of the reduction of latent heat of transformation, $\delta \mathrm{H}$, on the reduction in $\mathrm{T}_{0}$, assuming that that the entropy of the phases - i.e. the slope of the free energy curves - remains unchanged. 
constant, shear moduli) can be used to infer at least the sign of the change in $\mathrm{M}_{S}$ relative to stoichiometric NiTi, the calculations, when used in conjunction with the scarce available experimental data no strong trend is observed. It is indeed very likely that the transformation temperature is a complex function of the energy landscape (that also is temperature-dependent) around the austenite-martensite transformation path. While the work is still in progress, future efforts should focus on more concentrated alloys. The energy landscape as a function of alloying elements may also provide more insight into the observed alloying effects on the transformation behavior of NiTi-X alloys.

On the experimental side, more systematic studies that investigate the effects of composition not only on the mechanical properties of the austenite in NiTi-X but also on the enthalpy differences between austenite and martensite are necessary in order to make contact with calculations. While the work by Frenzel et al. [62] points to the right direction, other approaches, particularly highthroughput synthesis [65] and characterization may provide a more systematic investigation of the compositional effects on the thermodynamics, structure and transformation behavior in SMAs.

\section{Acknowledgments}

The authors would like to acknowledge the National Science Foundation (Grants No. DMR0844082 and DMR-0805293) for the support to complete this work. Partial support from NSFCMMI-0953984 and NSF-DMREF-1534534 is also acknowledged. First principle calculations were carried out in the Chemical Engineering cluster of Texas A\&M University, the Texas A\&M Supercomputing facility, and the Ranger and Lonestar clusters at the Texas Advanced Computing Center (TACC) in the University of Texas at Austin.

\section{References}

[1] J. Ma, I. Karaman, R. Noebe, High temperature shape memory alloys, International Materials Reviews 55 (5) (2010) 257-315.

[2] N. Morgan, Medical shape memory alloy applications-the market and its products, Materials Science and Engineering: A 378 (1) (2004) 16-23.

[3] M. Sanati, R. Albers, F. Pinski, Electronic and crystal structure of NiTi martensite, Physical Review B 58 (20) (1998) 13590.

[4] M. Carroll, C. Somsen, G. Eggeler, Multiple-step martensitic transformations in Ni-rich NiTi shape memory alloys, Scripta Materialia 50 (2) (2004) 187-192.

[5] W. Huang, On the selection of shape memory alloys for actuators, Materials \& Design 23 (1) (2002) 11-19.

[6] G. Song, D. Patil, C. Kocurek, J. Bartos, Applications of shape memory alloys in offshore oil and gas industry: a review, Proceedings of Earth and Space (2010) 14-17.

[7] R. Santamarta, R. Arróyave, J. Pons, A. Evirgen, I. Karaman, H. Karaca, R. Noebe, TEM study of structural and microstructural characteristics of a precipitate phase in Ni-rich $\mathrm{Ni}-\mathrm{Ti}-$ Hf and Ni-Ti-Zr shape memory alloys, Acta Materialia 61 (16) (2013) 6191-6206. 
[8] J. Cui, Y. S. Chu, O. O. Famodu, Y. Furuya, J. Hattrick-Simpers, R. D. James, A. Ludwig, S. Thienhaus, M. Wuttig, Z. Zhang, et al., Combinatorial search of thermoelastic shapememory alloys with extremely small hysteresis width, Nature Materials 5 (4) (2006) 286-290.

[9] K. Ng, H. Man, T. Yue, Corrosion and wear properties of laser surface modified NiTi with Mo and $\mathrm{ZrO}_{2}$, Applied Surface Science 254 (21) (2008) 6725-6730.

[10] Y. Shugo, F. Hasegawa, T. Honma, Effects of Copper addition on the martensitic transformation of TiNi alloy, Bull. Res. Inst. Min. Dressing Metall. Tohoku Univ. 37 (1) (1981) 79-88.

[11] O. Mercier, K. Melton, The substitution of $\mathrm{Cu}$ for $\mathrm{Ni}$ in NiTi shape memory alloys, Metallurgical and Materials Transactions A 10 (3) (1979) 387-389.

[12] S. Miyazaki, I. Shiota, K. Otsuka, H. Tamura, Effects of copper addition on mechanical behavior of Ti - Ni alloy, in: Proceedings of the MRS International Meeting on Advanced Materials., Vol. 9, 1988, pp. 153-158.

[13] T. Saburi, T. Takagaki, S. Nenno, K. Koshino, mechanical behavior of shape memory Ti - Ni $\mathrm{Cu}$ alloys, in: Proceedings of the MRS International Meeting on Advanced Materials., Vol. 9, 1988, pp. $147-152$.

[14] K. Eckelmeyer, The effect of alloying on the shape memory phenomenon in nitinol, Scripta Metallurgica 10 (8) (1976) 667-672.

[15] J. Mulder, J. Mass, J. Beyer, Martensitic transformation and shape memory effect in TiNiZr alloys, in: Proceedings of ICOMAT 1992, 1992, pp. 869-875.

[16] P. Potapov, A. Shelyakov, A. Gulyaev, E. Svistunov, N. Matveeva, D. Hodgson, Effect of Hf on the structure of Ni-Ti martensitic alloys, Materials Letters 32 (4) (1997) 247-250.

[17] E. Cesari, P. Ochin, R. Portier, V. Kolomytsev, Y. Koval, A. Pasko, V. Soolshenko, Structure and properties of $\mathrm{Ti}-\mathrm{Ni}-\mathrm{Zr}$ and $\mathrm{Ti}-\mathrm{Ni}-\mathrm{Hf}$ melt-spun ribbons, Materials Science and Engineering: A 273 (1999) 738-744.

[18] C. Wojcik, Properties and heat treatment of high transition temperature Ni-Ti-Hf alloys, Journal of Materials Engineering and Performance 18 (5) (2009) 511-516.

[19] E. Quandt, C. Halene, H. Holleck, K. Feit, M. Kohl, P. Schloßmacher, A. Skokan, K. Skrobanck, Sputter deposition of TiNi, TiNiPd and TiPd films displaying the two-way shape-memory effect, Sensors and Actuators A: Physical 53 (1) (1996) 434-439.

[20] N. Frantz, E. Dufour-Gergam, J. Grandchamp, A. Bosseboeuf, W. Seiler, G. Nouet, G. Catillon, Shape memory thin films with transition above room temperature from Ni-rich NiTi films, Sensors and Actuators A: Physical 99 (1) (2002) 59-63.

[21] H. Lin, K. Lin, S. Chang, C. Lin, A study of TiNiV ternary shape memory alloys, Journal of Alloys and Compounds 284 (1) (1999) 213-217.

[22] K. Oh, U. Joo, G. Park, C. Hwang, K. Kim, Effect of silver addition on the properties of nickel-titanium alloys for dental application, Journal of Biomedical Materials Research Part B: Applied Biomaterials 76 (2) (2006) 306-314. 
[23] Y. Koizumi, Y. Ro, S. Nakazawa, H. Harada, NiTi-base intermetallic alloys strengthened by Al substitution, Materials Science and Engineering: A 223 (1) (1997) 36-41.

[24] H. Hosoda, S. Hanada, K. Inoue, T. Fukui, Y. Mishima, T. Suzuki, Martensite transformation temperatures and mechanical properties of ternary NiTi alloys with offstoichiometric compositions, Intermetallics 6 (4) (1998) 291-301.

[25] A. Fasching, D. Norwich, T. Geiser, G. Paul, An evaluation of a NiTiCo alloy and its suitability for medical device applications, Journal of Materials Engineering and Performance 20 (4) (2011) 641-645.

[26] T. Duerig, A. Pelton, D. Stöckel, An overview of nitinol medical applications, Materials Science and Engineering: A 273 (1999) 149-160.

[27] K. Otsuka, X. Ren, Recent developments in the research of shape memory alloys, Intermetallics 7 (5) (1999) 511-528.

[28] T. Duerig, M. Wholey, A comparison of balloon-and self-expanding stents, Minimally Invasive Therapy \& Allied Technologies 11 (4) (2002) 173-178.

[29] C. P. G. R. B. Thurman, G. Bitton, The molecular mechanisms of copper and silver ion disinfection of bacteria and viruses, Critical Reviews in Environmental Science and Technology 18 (1989) 295-315.

[30] P. Bragg, D. Rainnie, The effect of silver ions on the respiratory chain of Escherichia coli, Canadian Journal of Microbiology 20 (6) (1974) 883-889.

[31] T. Nam, D. Chung, J. Kim, S. Kang, Phase transformation behaviors and shape memory characteristics of Ti-Ni-Mo alloys, Materials Letters 52 (3) (2002) 234-239.

[32] G. Bozzolo, R. Noebe, H. Mosca, Atomistic modeling of Pd site preference in NiTi, Journal of Alloys and Compounds 386 (1) (2005) 125-138.

[33] G. Bozzolo, H. Mosca, M. del Grosso, Energy of formation, lattice parameter and bulk modulus of $(\mathrm{Ni}, \mathrm{X}) \mathrm{Ti}$ alloys with $\mathrm{X}=\mathrm{Fe}, \mathrm{Pd}, \mathrm{Pt}, \mathrm{Au}, \mathrm{Al}, \mathrm{Cu}, \mathrm{Zr}, \mathrm{Hf}$, Intermetallics 16 (5) (2008) $668-675$.

[34] G. Bozzolo, R. D. Noebe, H. O. Mosca, Site preference of ternary alloying additions to NiTi: Fe, Pt, Pd, Au, Al, Cu, Zr and Hf, Journal of Alloys and Compounds 389 (1) (2005) 80-94.

[35] H. Mosca, G. Bozzolo, M. del Grosso, Atomistic modeling of ternary additions to NiTi and quaternary additions to Ni-Ti-Pd, Ni-Ti-Pt and Ni-Ti-Hf shape memory alloys, Physica B: Condensed Matter 407 (16) (2012) 3244-3247.

[36] D. Shin, R. Arróyave, Z.-K. Liu, A. Van de Walle, Thermodynamic properties of binary hcp solution phases from special quasirandom structures, Physical Review B 74 (2) (2006) 024204.

[37] A. Van de Walle, M. Asta, G. Ceder, The alloy theoretic automated toolkit: A user guide, Calphad 26 (4) (2002) 539-553. 
[38] A. Van de Walle, P. Tiwary, M. De Jong, D.L. Olmsted, M. Asta, A. Dick, D. Shin, Y. Wang, L.Q. Chen, Z.-K. Liu, Efficient stochastic generation of special quasirandom structures, Calphad 42 (2013) 13-18.

[39] K. Momma, F. Izumi, Vesta 3 for three-dimensional visualization of crystal, volumetric and morphology data, Journal of Applied Crystallography 44 (6) (2011) 1272-1276.

[40] P. Hohenberg, W. Kohn, Inhomogeneous electron gas, Physical Review 136 (3B) (1964) B864.

[41] J. Hafner, Materials simulations using VASP a quantum perspective to materials science, Computer Physics Communications 177 (1) (2007) 6-13.

[42] K. Burke, J. Perdew, Y. Wang, J. Dobson, G. Vignale, M. Das, Electronic density functional theory: Recent progress and new directions, Dobson, JF (1998) 81-112.

[43] P. Blöchl, Projector augmented-wave method, Physical Review B 50 (24) (1994) 17953.

[44] G. Kresse, D. Joubert, From ultrasoft pseudopotentials to the projector augmented-wave method, Physical Review B 59 (3) (1999) 1758.

[45] H. J. Monkhorst, J. D. Pack, Special points for Brillouin-zone integrations, Physical Review B $13(12)(1976) 5188$.

[46] M. Methfessel, A. Paxton, High-precision sampling for Brillouin-zone integration in metals, Physical Review B 40 (6) (1989) 3616.

[47] P. Blöchl, O. Jepsen, O. Andersen, Improved tetrahedron method for Brillouin-zone integrations, Physical Review B 49 (23) (1994) 16223.

[48] Y. Le Page, P. Saxe, Symmetry-general least-squares extraction of elastic data for strained materials from ab initio calculations of stress, Physical Review B 65 (10) (2002) 104104.

[49] T. Duong, S. Gibbons, R. Kinra, R. Arróyave, Ab-initio aprroach to the electronic, structural, elastic, and finite-temperature thermodynamic properties of Ti2AX $(\mathrm{A}=\mathrm{Al}$ or $\mathrm{Ga}$ and $\mathrm{X}=\mathrm{C}$ or N), Journal of Applied Physics 110 (9) (2011) 093504.

[50] O. Anderson, A simplified method for calculating the Debye temperature from elastic constants, Journal of Physics and Chemistry of Solids 24 (7) (1963) 909-917.

[51] R. Hill, The elastic behaviour of a crystalline aggregate, Proceedings of the Physical Society. Section A 65 (5) (2002) 349.

[52] H. Ding, B. Medasani, W. Chen, K. A. Persson, M. Haranczyk, M. Asta, PyDII: A python framework for computing equilibrium intrinsic point defect concentrations and extrinsic solute site preferences in intermetallic compounds, Computer Physics Communications 193 (2015) $118-123$.

[53] Y. Ye, C. Chan, K. Ho, Structural and electronic properties of the martensitic alloys TiNi, TiPd, and TiPt, Physical Review B 56 (7) (1997) 3678.

[54] X. Huang, C. Bungaro, V. Godlevsky, K. Rabe, Lattice instabilities of cubic NiTi from first principles, Physical Review B 65 (1) (2001) 014108. 
[55] D. Cheng, S. Zhao, S. Wang, H. Ye, First-principles study of the elastic properties and electronic structure of NiTi, CoTi and FeTi, Philosophical Magazine A 81 (6) (2001) 1625-1632.

[56] O. Mercier, K. Melton, G. Gremaud, J. Hägi, Single-crystal elastic constants of the equiatomic NiTi alloy near the martensitic transformation, Journal of Applied Physics 51 (3) (1980) 18331834 .

[57] D. Dunand, D. Mari, M. Bourke, J. Roberts, NiTi and NiTi-TiC composites: Part IV. Neutron diffraction study of twinning and shape-memory recovery, Metallurgical and Materials Transactions A 27 (9) (1996) 2820-2836.

[58] S. Rajagopalan, A. Little, M. Bourke, R. Vaidyanathan, Elastic modulus of shape-memory NiTi from in situ neutron diffraction during macroscopic loading, instrumented indentation, and extensometry, Applied Physics Letters 86 (8) (2005) 081901-081901.

[59] M.-X. Wagner, W. Windl, Lattice stability, elastic constants and macroscopic moduli of NiTi martensites from first principles, Acta Materialia 56 (20) (2008) 6232-6245.

[60] N. Hatcher, O. Y. Kontsevoi, A. Freeman, Role of elastic and shear stabilities in the martensitic transformation path of NiTi, Physical Review B 80 (14) (2009) 144203.

[61] Z.-Y. Zeng, C.-E. Hu, L.-C. Cai, X.-R. Chen, F.-Q. Jing, First-principles determination of the structure, elastic constant, phase diagram and thermodynamics of NiTi alloy, Physica B: Condensed Matter 405 (17) (2010) 3665-3672.

[62] J. Frenzel, A. Wieczorek, I. Opahle, B. Maaß, R. Drautz, G. Eggeler, On the effect of alloy composition on martensite start temperatures and latent heats in Ni-Ti-based shape memory alloys, Acta Materialia 90 (2015) 213-231.

[63] X. B. Ren, K. Otsuka, Why does the martensitic transformation temperature strongly depend on composition?, Materials Science Forum, Vol. 327, Trans Tech Publ, 2000, pp. 429-432.

[64] M. Zarinejad, Y. Liu, Dependence of transformation temperatures of NiTi-based shape memory alloys on the number and concentration of valence electrons, Advanced Functional Materials 18 (18) (2008) 2789-2794.

[65] P. J. McCluskey, K. Xiao, J. M. Gregoire, D. Dale, J. J. Vlassak, Application of in-situ nanoscanning calorimetry and x-ray diffraction to characterize $\mathrm{Ni}-\mathrm{Ti}-\mathrm{Hf}$ high-temperature shape memory alloys, Thermochimica Acta 603 (2015) 53-62. 